CUAD. CONTAB. / BOGOTÁ, COLOMBIA, 15 (39): 787-805 / NÚMERO ESPECIAL 2014 / 787

\title{
Tendencias en contabilidad de gestión: una mirada a su evolución (finales del siglo $\mathrm{XIX}$ y siglo $\mathrm{XX}$ )
}

doi: 10.11144/Javeriana.cc15-39.tcgm

\section{Jorge Andrés Salgado-Castillo}

Contador público, Universidad del Valle (Buga). Candidato

a magíster en gestión de organizaciones, Universidad

Militar Nueva Granada (Bogotá). Docente de tiempo

completo, Facultad de Ciencias Económicas, Universidad

Militar Nueva Granada (programa de Contaduría Pública).

Integrante del Grupo de Estudios Contemporáneos en

Contabilidad, Gestión y Organizaciones, Universidad

Militar Nueva Granada.

Correos electrónicos: jorge.salgado@unimilitar.edu.co, jascx12@gmail.com. Twitter: @JorgeASalgadoC,

Skype: jascuninuevagranada

\footnotetext{
* El presente texto se deriva de las etapas conceptuales de los siguientes proyectos de investigación: Tendencias de investigación en Contabilidad de Gestión en Iberoamérica (1998-2008). Proyecto ECO-563 (vigencia 2010); Influencia de los sistemas de control de gestión sobre el desempeño organizacional: caso empresas industriales del grupo social y empresarial de la defensa (GSED). Proyecto ECO-1487 (vigencia 2014), financiados por la Vicerrectoría de Investigaciones de la Universidad Militar Nueva Granada.
} 
Resumen La contabilidad de gestión es considerada como un componente importante de la división disciplinar de la contabilidad. Aquella está influenciada por por contextos económicos y sociales y se inserta en un marco temporal que la configura. Estos tiempos y contextos particulares influyen y son influidos por el significado, el sentido y las prácticas contables. El recorrido por algunos hitos del desarrollo que conforman la evolución en la contabilidad gerencial en el ámbito internacional, se puede agrupar en tendencias que han sido objeto de reflexión académica y de aplicación en las unidades empresariales. Con un enfoque descriptivo, se pretende mostrar los recorridos desde una visión de lo histórico, y lo académico investigativo, para resaltar los principales aportes en ambos campos. El nacimiento de la contabilidad de gestión se muestra desde los entornos organizacionales de producción, que cada vez más son objeto de mayor reflexión e investigación, que se ha establecido en la actualidad como componente fundamental del conocimiento disciplinar contable.

Palabras clave contabilidad de gestión; tendencias; internacional; evolución; contribuciones; desarrollo académico; investigación

\section{Código JEL M 41}

Tendencies on Management Accounting:

\section{A Look at their Evolution (Late XIX Century and $\mathrm{XX}$ Century)}

\footnotetext{
Abstract Management accounting is considered as a branch of the accounting discipline influenced by social and economic contexts; it is the branch inserted in a time framework that configures it. These particular times and contexts influence and are influenced by the meaning, sense, and practices of accounting. By following the road of some development milestones that are part of the evolution of management accounting in the international sector, we can group them into tendencies that have been the subject
}

matter of academic reflection and application in corporate units. Our aim is to show these roads, with a descriptive focus, from a historical and academic-research point of view, in order to highlight the main contributions to both fields. The origin of management accounting is shown from the organizational production environments - these are subject to greater reflection and research with increasing frequency - and it currently is a fundamental component of the accounting discipline knowledge.

Keywords management accounting; tendencies; international; evolution; contributions; academic development; research

\section{Tendências em contabilidade de gestão: um olhar para sua evolução (finais do século $X I X$ e século $X X$ )}

Resumo A contabilidade de gestão é considerada como um ramo da disciplina contábil, influenciada por contextos económicos e sociais; aquela insere-se num marco temporal que a configura. Estes tempos e contextos particulares influenciam e são influenciados pelo significado, o sentido e as práticas contábeis. O percurso por alguns marcos miliários do desenvolvimento que conformam a evolução na contabilidade gerencial na arena internacional, podese agrupar em tendências que já foram objeto de reflexão académica e aplicação nas unidades empresariais. Com enfoque descritivo, visa-se mostrar os percursos desde uma visão do histórico e o acadêmico investigativo, para remarcar as principais contribuições em ambos os campos. O nascimento da contabilidade de gestão é mostrado desde os entornos organizacionais de produção, que cada vez mais são objeto de mais reflexão e pesquisa, estabelecido na atualidade como componente fundamental do conhecimento disciplinar contábil.

Palavras-chave contabilidade de gestão; tendências; internacional; evolução; contribuições; desenvolvimento académico; pesquisa 


\section{Introducción}

Los cambios en el entorno económico, los cambios sociales, culturales, tecnológicos, ambientales, entre otros, influyen en la contabilidad de gestión. Algunos autores (Carmona, 1993; Johnson \& Kaplan, 1988; Kaplan, 1988; Lunkes, Ripoll-Feliu \& Silva da Rosa, 2012) han resaltado que en la última mitad del siglo XIX y el primer cuarto del siglo XX, algunas empresas gozaban de cierta estabilidad en sus actividades productivas y de gestión, y ponían poca atención a las variables ambientales, por su presunta poca vulnerabilidad. Un cambio importante se da desde mediados de los años setenta del siglo XX, cuando los sistemas productivos empresariales empezaron a evolucionar. Los entornos económicos han pasado a ser dinámicos, complejos, turbulentos y globalizados, y han emergido fenómenos económicos, sociales y culturales, como las crisis financieras pronunciadas, los avances tecnológicos, las culturas interconectadas, etc. Los tiempos actuales se relacionan con contextos y realidades diferentes a los del siglo XX; entre otros fenómenos, están la expansión de la tecnología de la información, una competencia global, una reducción del ciclo de vida de los productos, la emergencia de la sociedad del conocimiento, las innovaciones tecnológicas y organizacionales.

La gestión de las empresas tiende a adaptarse a la realidad económica, o más bien está influenciada por ella ${ }^{1}$. Un ejemplo de lo anterior

1 De acuerdo con Anthony G. Hopwood (1987) y Fernando Gutiérrez-Hidalgo (2005), se puede afirmar que la contabilidad de gestión está, al igual que otras realidades de la em- es lo planteado por José Álvarez-López y Felipe Blanco-Ibarra (2003), con la transformación de sistemas presupuestarios en organizaciones con un crecimiento y estabilidad lineal, y en modelos de planificación estratégica en épocas de crisis, por la necesidad de afrontarlas de manera adecuada. Lo anterior genera cambios cada vez más notorios en la contabilidad de gestión, que se ha enfrentado a los requerimientos organizacionales y a su necesidad de adaptación y de conocer la información adecuada para la toma de decisiones en estos nuevos contextos, así que las organizaciones buscan herramientas apropiadas de gestión que contribuyan a adaptar los cambios en las mejores condiciones posibles (Álvarez \& Blanco, 2003). Estos fenómenos plantean un escenario para que los sistemas de información internos guíen a las empresas a tomar decisiones de inversión, a fijar objetivos a largo y a corto plazo y a medir la eficiencia de sus procesos y la rentabilidad de sus productos de acuerdo a las necesidades cambiantes (Gutiérrez, 2005).

El presente texto pretende describir las tendencias internacionales en contabilidad de gestión que han tenido influencia en las organizaciones y que han sido objeto de investigación y reflexión académica. Se quiere mostrar además la importancia de la contabilidad de gestión y su evolución no solo desde una perspectiva que evidencie el impacto que ha tenido en las empresas, sino también en las temáticas importantes en la producción académica internacional. La estructura del documento se di-

presa, insertada en su entorno y que, en un doble sentido, es configurada por él y es capaz de configurarlo al mismo tiempo. 
vide en tres apartados, además de la presente introducción, la discusión y las conclusiones. Inicialmente, se esboza un recorrido histórico que resalta la aparición y desarrollo de ciertas prácticas y temáticas en contabilidad de costos y gestión de acuerdo a sus entornos particulares, este apartado pretende mostrar algunas contribuciones hechas a finales del siglo XIX y siglo XX, fundamentalmente apoyados en Joseph-H. Vlaemminck (1961) y Robert S. Kaplan (1984, 1993). Posteriormente, se muestran algunas tendencias que permiten vislumbrar el desarrollo académico en la disciplina en la segunda mitad del siglo XX; este punto se desarrolla a partir del aporte de Richard Mattessich (2001). En el tercer apartado, se muestran los enfoques, corrientes, métodos y temáticas internacionales de investigación en contabilidad de gestión en el siglo XX, se hace una referencia a la investigación mundial, iberoamericana y se da una pequeña reflexión en torno al caso colombiano, se tomarán principalmente los aportes de Bob Ryan, Robert W. Scapens y Michael Theobald (2004), Bernabé Escobar-Pérez y Antonio Lobo-Gallardo (2002), James W. Hesford, Sung-Han Lee, Wim A. van der Stede y S. Mark Young (2007) y Rogério João Lunkes, Vicente Ripoll-Feliu y Fabricia Silva da Rosa (2012). Al final, las conclusiones.

\section{Tendencias en contabilidad de gestión: descripción de su surgimiento y evolución en el contexto anglosajón dominante}

De acuerdo con Joseph-H. Vlaemminck (1961), dentro del plan de una síntesis histórica o de una elección de hechos en determinados períodos, no es posible hacer mención de todos los autores y todas las tendencias que se han ido manifestando en un período dado. Se hace preciso, pues, proceder a una selección quenecesariamente- estará llena de lagunas (Vlaemminck, 1961) y es arbitraria; por tanto, no es completa y concluyente. La historia de la contabilidad es una disciplina que abarca una amplitud temporal considerable, ya que como actividad es tan antigua como la propia humanidad.

"Desde que el hombre es hombre, y aun mucho antes de conocer la escritura, ha necesitado llevar cuenta, guardar memoria y dejar constancia de datos relativos a su vida económica y a su patrimonio" (Hernández, 2006, p. 193). La historia de la contabilidad de costos y de gestión se remonta a orígenes antiguos y su seguimiento en todos los tiempos no es la tarea del presente apartado. La intencionalidad principal reside en mostrar algunas temáticas y tendencias con su consecuente surgimiento atribuido a períodos explícitos; en este caso, se plantean ciertas temáticas surgidas a finales del siglo XIX y contribuciones en el siglo XX.

De acuerdo con Kaplan $(1984,1993)$, la demanda de información para la planificación interna y el control surgió aparentemente en la primera mitad del siglo XIX, cuando empresas como las fábricas textiles y los ferrocarriles tuvieron que idear procedimientos administrativos internos para coordinar los múltiples procesos inmersos en su actividad principal. Citando a H. Thomas Johnson (1972), Robert S. Kaplan $(1984,1993)$ muestra el caso de Lyman Mills, un molino textil de Nueva Inglaterra²,

2 Fundado aproximadamente en 1855. 
cuyo sistema de contabilidad estaba basado en los libros por partida doble, y daba información acerca del costo del producto terminado, de la productividad laboral, del impacto de los cambios en la distribución de la planta y del control de la recepción y uso del algodón. En el caso de las empresas de ferrocarriles (Chandler, 1977; Kaplan, 1984, 1993), se muestra la generación de informes financieros resumidos sobre las operaciones de muchas unidades grandes y dispersas geográficamente; también se desarrolló un sistema estadístico que daba cuenta del costo por tonelada y milla y la ratio de operación ${ }^{3}$, que eran rutinariamente calculados para las diferentes subunidades y clases de servicio. Los sistemas contables de los ferrocarriles se trasladaron posteriormente a las organizaciones de producción en masa. Un caso emblemático es el de la empresa de acero de Andrew Carnegie en 1880: el director general William P. Shinn enviaba a Carnegie informes mensuales, incluso diarios, con datos sobre los costos del mineral, la piedra caliza y el carbón, entre otros materiales y mano de obra por tonelada de rieles producida; los más mínimos detalles del costo de los materiales y de la mano de obra de cada departamento aparecían día tras día y semana a semana en las cuentas (Chandler, 1977; Kaplan, 1984, 1993). Carnegie se enfocó en sus costos y se aseguró de tener los costos más bajos de la industria, esto le dio una gran ventaja ya que se encargó de hacer reducciones de precios durante la recesión económica, "mientras que el resto de empresas sufrían pérdidas, él aún generaba beneficios" (Johnson, 1981; Kaplan, 1984, 1993, p. 59).

3 Gastos de explotación divididos por las ventas.
A comienzos del siglo XX, en la etapa de la administración científica, la industria estadounidense dio un mayor ímpetu al desarrollo de las prácticas de contabilidad de costos impulsadas por directivos e ingenieros (Chandler, 1977; Kaplan, 1984, 1993). Entre varias temáticas, se puede resaltar como innovación, la determinación de los costos indirectos de fabricación y la asignación tanto de los costos directos como los indirectos a cada uno de los productos fabricados; el desarrollo de los costos estándar también se le atribuye al período comprendido entre 1908 y 1918. En esta etapa, los costos estándar evolucionaron hasta llegar a la práctica en las empresas. En adición a lo anterior, se usaron los gráficos de punto de equilibrio ${ }^{4}$ para expresar la desviación de los costos con el output, lo que está documentalmente probado en Inglaterra y Estados Unidos en 1903 y 1904 (Solomons, 1968; Kaplan, 1984, 1993).

Joseph-H. Vlaemminck (1961) relaciona la influencia que tuvo la administración científica en la contabilidad desde los años 1940 en adelante. De manera más específica, la obra Administración industrial y general ${ }^{5}$, de Henri Fayol (1961), establece seis grupos de operaciones o funciones esenciales que existen en la empresa; una de aquellas funciones es la función de contabilidad, que define: "el órgano de visión de la empresa debe permitir conocer en todo instante dónde se está y adónde se va. Debe dar sobre la situación económica de la empresa informes exactos, claros, precisos. Una buena contabilidad, sencilla y clara, que permite tener una

\footnotetext{
4 También denominados gráficos de punto muerto. 5 Traducción de Administration industrielle et générale. Paris: Dunod (1941).
} 
idea exacta de las condiciones de la empresa, es un medio poderoso de dirección" (Fayol, 1961, p. 135). Este aspecto no es menor, ya que da cuenta de la relación importante entre administración y contabilidad, que da sustento en gran medida a la contabilidad de gestión. En este sentido, se establece "que los vínculos cada vez más profundos entre contabilidad y la organización de empresas exigen por parte de los contadores conocimientos cada vez más profundos en materia de organización científica" (Vlaemminck, 1961).

Joseph-H. Vlaemminck (1961) resalta el hecho de la relación contabilidad y administración empresarial, citando a Urbain Vaes (1952) anota que la organización de la contabilidad debe necesariamente adaptarse a la organización general, de la que constituye uno de los mejores instrumentos de coordinación y control (Vlaemminck, 1961, p. 361). Enfoca también la "contabilidad industrial" más como un instrumento de gestión que de registro, y que sirve a los intereses de la empresa. También se pone de relieve el uso de la contabilidad presupuestaria que pone especial interés en determinar el costo de las diversas actividades para ayudar a los directivos a reducir sus costos (Dumortier, 1952, citado por Vlaemminck, 1961), uno de los más destacados avances de los métodos de dirección y de administración logrados en el segundo cuarto del siglo XX ha sido la adopción del sistema de control presupuestario por un número creciente de grandes empresas y de organismos públicos (Pasdermadjian, 1947, citado por Vlaemminck, 1961). En general, Joseph-H. Vlaemmninck (1961) anota que la economía de empresa y la contabilidad in- dustrial son conocimientos fundamentales en la empresa y que:

\begin{abstract}
Se requieren componentes asociados a los problemas comunes a todas las empresas (principios de dirección científica, gestión y control, gestión de salarios y relaciones humanas del trabajo, administración de personal y política social, financiación y política financiera, métodos de análisis del rendimiento y de la estabilidad, costos y política industrial, ventas y política de ventas, análisis de mercados, técnica administrativa, mecanización del trabajo administrativo, etc.) (...) existe una influencia notable de la técnica de la contabilidad sobre el desarrollo de los métodos de dirección y de análisis de rendimiento (Vaes, 1952, citado por Vlaemminck, 1961, p. 366).
\end{abstract}

En lo referente al control de gestión, en 1910, la Dupont Powder Company utilizaba casi todos los métodos básicos que se usan actualmente en la gestión de una empresa (Chandler, 1977; Kaplan, 1984, 1993). Esta organización descentralizada funcional (organización multiproducto integrada verticalmente) requería un sistema de medida del desempeño para motivar y evaluar los resultados de cada departamento y, para guiar la estrategia de la empresa. Dupont innovó ${ }^{6}$ el cálculo del Retorno sobre la Inversión (return on investment, ROI) que sirvió como indicador de eficiencia departamental y como medida del rendimiento de la empresa en su conjunto. De acuerdo con Robert S. Kaplan (1984), el desarrollo del ROI en cada departamento parece ser el origen de los con-

6 Algunos autores lo denominan "invención”. 
ceptos de centros de beneficio y de inversión. A principio de 1920, Dupont tomó el control de General Motors como principal accionista ante las dificultades financieras, y estableció ciertos cambios, entre los cuales se destacan los niveles satisfactorios de retorno sobre las inversiones en un ciclo completo de negocios, en contraposición a los incrementos de utilidades anuales, el establecimiento de un sistema de incentivos para otorgar recompensas a aquellos empleados y directivos que hicieran contribuciones sustanciales al resultado de la empresa, y un sofisticado sistema de precios de transferencia entre las divisiones de General Motors; este concepto ya se había aplicado como los anteriores en Dupont en 1905 (Kaplan, 1984). Con respecto a estas dos organizaciones, Kaplan concluye:

\begin{abstract}
Hacia 1925, Dupont y General Motors habían desarrollado muchas de las prácticas de control de gestión: la descentralización vía organización funcional o multidivisional, el ROI como medida del rendimiento, los procedimientos formales de asignación de capital, los ciclos presupuestarios y planificación, los presupuestos flexibles, la fijación de precios según el ROI y el volumen estándar, los planes de incentivos y de participación en beneficios, y una política de precios de transferencia basada en los precios del mercado (Kaplan, 1984, 1993, p. 70).
\end{abstract}

La contabilidad de costos ha ido reformulando y mejorando sus técnicas. J. Hugh Jackson (1979) muestra que el período comprendido entre 1920 y 1945 fue una etapa de crecimiento en la disciplina; se dieron mejo- rías en las técnicas de cálculo de costos, en la amplitud de sus utilizaciones a prácticamente todos los negocios; se contempló la elevación y refinamiento de los costos estándar y, en un menor grado, el presupuesto flexible. Durante este período, continuó la aceptación por parte de la administración, que reconocía el valor de los métodos científicos de contabilidad de costos (Jackson, 1979), no solo como medio de control de costos, sino también como medio de reducirlos. La administración se dio cuenta de que para fundamentar sus políticas y sus planes, necesitaba los datos que los métodos de cálculo de costos generaban.

Después de 1925 y hasta 1980 se han hecho contribuciones a la contabilidad de costos y gestión. De acuerdo con Kaplan $(1984,1993)$ la diferencia con las contribuciones anteriores estriba en la casi nula innovación por parte de los directivos o contables de gestión, su consecuente poco impacto sobre el pensamiento contable de gestión contemporáneo y la abundante actividad académica pero con poca trascendencia en la práctica. Prácticamente, todas las técnicas empleadas en las empresas en los años ochenta y explicadas en los principales libros de costos, ya habían sido desarrolladas en 1925. A pesar del cambio considerable en las organizaciones y en la dimensión de la competencia, ha habido poca o nula innovación en el diseño e implementación de los sistemas de contabilidad de costos y de los sistemas de control de gestión (Kaplan, 1984, 1988, 1993). Las necesidades actuales no pueden ser afrontadas con prácticas de contabilidad de costos desarrolladas en tiempos y contextos diferentes a los actuales (Kaplan, 1984, 1993). 
En los años cincuenta, se destacan las temáticas de los flujos de caja descontados frente al ROI, y el ingreso residual atribuido a desarrollos realizados en la empresa General Electric. En los años sesenta, sobresale la corriente en contabilidad de gestión estimulada por los desarrollos de la investigación operativa, cuyo centro estuvo en la utilización de modelos cuantitativos para una variedad de problemas de costeo, control y planificación. De 1965 a 1980, se aplica la economía de la información, la teoría de la agencia y el modelo de los costos de transacción a los problemas de la Contabilidad de gestión.

\section{Tendencias en contabilidad de gestión: un vistazo al desarrollo académico en la segunda mitad del siglo $X X$}

La década de los cincuenta del siglo pasado constituyó la transición del enfoque tradicional contable hacia un enfoque "más científico", con prevalencia de elementos provenientes de las matemáticas modernas y las ciencias sociales (Mattessich, 2001). Se destacan los estudios econométricos del costo por parte de economistas de dirección como Joel Dean (1951), citado por Richard Mattessich (2001), las contribuciones de los expertos en investigación operativa a la contabilidad de costos y gestión como Abraham Charnes y William W. Cooper $(1957,1961)$, citados por Richard Mattessich (2001).

En los años sesenta se continuó con el interés en temáticas como los puntos de equilibrio, las relaciones costo-volumen y el análisis de varianzas; adicional a esto, se realizaron contribuciones a los temas de costo marginal frente al costo completo, costos relevantes y precios de transferencia (Mattessich, 2001). Otra tendencia que se avizoraba en los años sesenta fue la influencia de la teoría financiera en la contabilidad y la necesidad de una investigación empírica; la búsqueda de métodos estadístico-empíricos recayó primero en las finanzas pero pasó de manera rápida a la contabilidad (Mattessich, 2001). En los años setenta, la investigación contable empírica por un lado y la investigación de información de agencia ${ }^{7}$ por el otro, empezaron a dominar la contabilidad académica por lo menos en Estados Unidos y asumieron en este período la categoría de ciencia normal. La contabilidad empírica comenzó a difundirse rápidamente en los países de habla inglesa, pero mucho más despacio en el resto. A finales de los años setenta del siglo XX, se marcó un hito con la publicación de los textos que dan cuenta de la "teoría de la contabilidad positiva" que potenció de manera importante la investigación empírica de corte positivo con el aporte de Ross L. Watts y Jerold Zimmerman (1978, 1979), la investigación contable empírica comprende muchos aspectos y generó una gran cantidad de publicaciones. Otra dirección surgió en Gran Bretaña con el enfoque que privilegia los aspectos sociales, organizacionales y críticos de la disciplina contable ${ }^{8}$.

En los años ochenta del siglo XX, las publicaciones de contabilidad académica de habla inglesa aumentaron en más del triple y, por tanto, la difusión se acrecentó de manera con-

\footnotetext{
7 Muchos estudios trataban de la relación contractual entre el principal y el agente y los costos de agencia. 8 Asociada con las publicaciones Accounting, Organizations and Society y Critical Perspectives in Accounting.
} 
siderable. Richard Mattessich (2001), entre otros factores, lo atribuye a un cambio generacional en la comunidad académica contable y el aumento de la oferta educativa contable. La investigación contable empírica continuó aflorando en muchos estudios con el impulso adicional del libro Positive Accounting Theory, de Ross L. Watts y Jerold Zimmerman (1986). Las perspectivas interpretativas siguen creciendo y tienen como referente a la sociología y a la filosofía; al mismo tiempo, la perspectiva crítica con el impulso de un grupo pequeño de contadores con tradición marxista (Mattessich, 2001; Ryan, Scapens \& Theobald, 2002).

La influencia de la teoría financiera fomentó en gran medida la investigación en contabilidad financiera, mientras que otras áreas — como la contabilidad de gestión— estaban descuidadas (Mattessich, 2001). Sin embargo, en los años ochenta y noventa se prestó una renovada atención mundial a los aspectos más prácticos de la contabilidad de gestión; la obra Relevance Lost - The Rise and Fall of Management Accounting, de H. Thomas Johnson y Robert S. Kaplan (1987) dio un empuje especial a la contabilidad de gestión. Este resurgimiento de la contabilidad de gestión se reflejó en nuevos conceptos y revivieron conceptos anteriores (dirección de costos estratégicos y contabilidad de actividades, entre otros); los trabajos publicados empezaron a aumentar de manera considerable, una metodología como el estudio de caso resurgió impulsada principalmente por Kaplan y por la Harvard Business School. Esto provocó un enfoque hacia la llamada investigación basada en el campo y en la interacción entre investigación y acercamiento a las orga- nizaciones. Este enfoque expuso un interés por mostrar varios temas en estadios más maduros o incluso nuevos; sin embargo, había una preocupación por la aplicabilidad y la verdadera innovación en los conceptos. George Foster y S. Mark Young (1997, pp. 75-76) citados por Richard Mattessich (2001) expresaron:

$$
\begin{aligned}
& \text { La mayoría de la literatura sobre contabili- } \\
& \text { dad de gestión, en un sentido amplio, trata } \\
& \text { sobre propuestas para nuevos sistemas de } \\
& \text { costos, nuevos sistemas de evaluación del } \\
& \text { rendimiento, nuevos sistemas de presupues- } \\
& \text { tos, etc. Es típico que se considere que los } \\
& \text { sistemas propuestos son mejores que los sis- } \\
& \text { temas ya existentes. Lo sorprendente es que } \\
& \text { en la literatura de investigación no haya un } \\
& \text { análisis sistemático de lo que significa mejor, } \\
& \text { cómo sería la forma correcta de medirlo y } \\
& \text { qué desafíos se encuentran cuando se reali- } \\
& \text { cen estas mediciones... }
\end{aligned}
$$

Sin duda, el período comprendido entre 1981 y 2000 tuvo avances importantes para la Contabilidad de Gestión. H. Thomas Johnson y Robert S. Kaplan (1988) influenciaron una corriente de trabajo importante y mostraron en su Auge y caída de la contabilidad de gestión varios problemas de la disciplina. En la década de los noventa, la contabilidad de gestión muestra horizontes y planteamientos que se pueden vislumbrar en las temáticas de sistemas de costos por actividad $(\mathrm{ABC})$; el desarrollo de los planteamientos del control de la calidad total (TQM) en el ámbito de la contabilidad de gestión; el control contable de la calidad, los sistemas de planificación de las necesidades de materiales (MRP), las tecnologías 
de producción óptima, las técnicas justo a tiempo (Just in time, JIT), los sistemas de fabricación flexible, la contabilidad de gestión medioambiental, el cuadro de mando integral o Balanced Scorecard (CMI/BSC), entre otros (Ripoll-Feliu, 1994, citado por Gutiérrez-Hidalgo, 2005).

En esta etapa, la contabilidad de gestión continuó tratando temas tradicionales como los precios de transferencia y las relaciones costovolumen, y las aplicaciones de la teoría de la información y teoría de la agencia en contabilidad de gestión (Mattessich, 2001).

En la segunda mitad del siglo XX, Richard Mattessich (2001) resalta el aumento en la sofisticación, diversificación y volumen de las publicaciones. La sofisticación está dada por la adaptación de métodos y técnicas prestadas de otras disciplinas, sobre todo de las ciencias matemáticas, la investigación operativa, la economía, las finanzas, el comportamiento, el derecho, la educación, la sociología, la filosofía, la educación, la historia y la arqueología. En lo referente a la diversificación y el volumen de publicaciones, se le atribuye a la tasa de crecimiento de la profesión contable; de ahí que haya más estudiantes, profesores e investigadores de contabilidad con una consecuente mayor actividad investigativa y publicaciones.

\section{Tendencias en contabilidad de gestión: enfoques, corrientes, métodos y temáticas de investigación internacional, siglo $\mathrm{XX}$}

Bob Ryan, Robert W. Scapens y Michael Theobald (2004) identifican las corrientes de investigación en contabilidad de gestión como tradiciones de investigación. La principal agrupación dentro de estas tradiciones de investigación está dada por la corriente dominante basada en la economía y las tradiciones alternativas en contabilidad de gestión. En la corriente principal basada en la economía, resaltan el paso del enfoque normativo al enfoque positivo, la influencia de la economía neoclásica, la economía de la información y la teoría de agencia en la contabilidad de gestión, y los presupuestos basados en los conceptos de racionalidad y comportamiento económico. Esta corriente ubicada en un enfoque funcionalista (Facin-Lavarda, Barrachina \& Ripoll-Feliu, 2008; Larrinaga-González, 1999; Lukka, 2010) permitió el desarrollo de técnicas y herramientas que son las características principales de la mayoría de los textos sobre contabilidad de gestión. Entre las tradiciones alternativas en la investigación en contabilidad de gestión, se destacan la investigación de la contabilidad conductista o conductual, el impacto de la teoría de la organización, la teoría social (disciplina sociológica), los enfoques interpretativos, el impacto de la teoría crítica y la investigación orientada a la práctica (Ryan, Scapens \& Theobald, 2004). En general, sobresale la diversidad de enfoques utilizados en la investigación en contabilidad de gestión; estos enfoques son variados y coexisten, lo que evidencia las asunciones filosóficas con respecto al conocimiento, a la manera de obtenerlo y a su validación (Chua, 1986; Larrinaga-González, 1999).

\section{Bernabé Escobar-Pérez y Antonio Lobo-} Gallardo (2002) hacen un recorrido por la evolución de los enfoques teóricos en contabilidad 
de gestión. Estos enfoques se han ido reformulando desde las aproximaciones neoclásicas que concebían la contabilidad de gestión como un conjunto de técnicas de modelización del comportamiento de cara a la consecución de los objetivos de la firma. En esta época dominada por el racionalismo económico, se introducen la incertidumbre y el valor de la información en la toma de decisiones; aparecen entonces los modelos de la decisión en contabilidad de gestión, las formulaciones inspiradas en la teoría estadística de la decisión y en la economía de la información; este enfoque puede rastrearse desde los años 50 y 60 del siglo XX. Posteriormente, en los años 70, la consideración de los contextos en el diseño de los sistemas contables es un aporte de los postulados de la teoría contingente y de la teoría organizacional en la contabilidad de gestión. En los años 80, la preocupación por los aspectos sociales y políticos provocó la entrada de una serie de planteamientos procedentes de la sociología interpretativa y de la psicología; se destaca la teoría institucional. Posteriormente, incluso en la actualidad, se están aplicando enfoques sociológicos en la investigación en contabilidad de gestión; sobresalen el institucionalismo sociológico y económico, las derivaciones de la sociología interpretativa y la teoría crítica (Escobar \& Lobo, 2002). Todas las corrientes teóricas han coexistido e incluso coexisten en la actualidad en distinto grado.

Los métodos de investigación de corte económico y contingente comenzaron a ser cuestionados y utilizados en menor medida; se puede apreciar una creciente importancia de los métodos cualitativos, posiblemente, por su capacidad para captar la forma en la que operan ciertos fenómenos en su contexto y que tienen un componente social y político, de cara al análisis de las prácticas tal como se producen en las organizaciones, normalmente caracterizadas por el dinamismo y por una elevada incertidumbre (Escobar \& Lobo, 2002). Entre los métodos cualitativos, se destaca el estudio de caso como una estrategia de investigación flexible y capaz de englobar varias técnicas de recolección de evidencia — como el análisis documental, las entrevistas y las encuestas-, por lo cual permite la triangulación metodológica.

James W. Hesford, Sung-Han Lee, Wim A. van der Stede y S. Mark Young (2007) muestran las tendencias más importantes en contabilidad de gestión desde 1981 hasta 2000. En este período hubo muchos avances en el campo pues nacieron o florecieron el costeo basado en actividades $(\mathrm{ABC})$, la contabilidad de gestión japonesa, la contabilidad de gestión estratégica y control, el cuadro de mando integral o Balanced Scorecard, entre otros tópicos (Hesford, Lee, Stede \& Young, 2007; Salgado, 2011). El control de gestión ocupa el lugar principal con bastante distancia sobre las otras temáticas; en esta temática, se destacan los sistemas de control en las organizaciones o control organizacional como los sistemas de control de gestión; también se destacan la medición y evaluación del desempeño y los presupuestos. El traslado de la contabilidad de costos hacia la contabilidad de gestión se materializa en los estudios e investigaciones realizadas; sin embargo, los costos continúan vigentes en la actualidad. Aunque en menor medida, los temas más relevantes son los centrados en la asignación de gastos genera- 


\section{8}

les y conjuntos, el análisis de factores o inductores de costo, el costeo basado en actividades y los costos basados en la capacidad. La información de costos para la toma de decisiones, el estudio de las varianzas de costos y el análisis de sistemas de contabilidad de costos en países específicos también hacen parte de los temas de estudio. Otras temáticas internacionales trabajadas en menor medida son los precios de transferencia, los que documentan o proponen métodos de investigación, la gestión estratégica y la gestión de la calidad total (TQM).

Los investigadores en contabilidad de gestión se acercan de maneras diversas a los problemas de investigación que plasman en sus investigaciones; no hay un método que aventaje a los demás de acuerdo a las publicaciones. Los métodos de investigación que podemos destacar, de acuerdo con James W. Hesford, Sung-Han Lee, Wim A. van der Stede y S. Mark Young (2007), son en su orden de importancia, los que desarrollan marcos conceptuales que proporcionan nuevas perspectivas ( $\mathrm{fra-}$ meworks). Los métodos analíticos aplicados a la contabilidad empírica y formal con énfasis en técnicas analíticas con utilización importante de la estadística, las investigaciones basadas en encuestas (survey) que especifican relaciones causales con fundamentos conceptuales en las que se remarca la necesidad de una buena teoría, la aplicación de encuestas por correo, teléfono, correo electrónico, internet o encuestas cara a cara, aunque todavía hay poca literatura contable con referencia a las encuestas por internet. El método experimental en contabilidad de gestión se basa en datos recogidos por el investigador para darles diversos tratamientos; la asignación puede ser aleatoria y se intenta manipular una variable. Otros métodos importantes en la contabilidad de gestión actual son los estudios de caso y los estudios de campo que estudian respectivamente, fenómenos contemporáneos que pueden incluir las personas, los procedimientos y las estructuras en una o en más organizaciones. En menor medida, se utilizan los métodos de archivo o análisis documental (archival) y revisión (review).

Las disciplinas de referencia en los estudios de contabilidad de gestión muestran la economía como principal referente de la contabilidad de gestión. Esto corrobora que prevalece la corriente de la contabilidad basada en la economía de corte positivo, en especial los referentes relacionados con la organización industrial, la microeconomía y la teoría de la agencia. Sin embargo, la sociología ha ganado un espacio en contabilidad de gestión y casi ha igualado la influencia de la economía; se destacan la teoría organizacional, la teoría de la contingencia y la teoría institucional. Otra disciplina de influencia importante es la psicología que cubre la psicología social, la psicología cognitiva y el comportamiento organizacional.

Rogério João Lunkes, Vicente Ripoll-Feliu y Fabricia Silva da Rosa (2012) estudiaron artículos en lengua española desde 2001 hasta 2010; su objetivo era evaluar la producción académica y compararla con las tendencias internacionales (Hesford, Lee, Stede \& Young, 2007). Los resultados muestran que la contabilidad de gestión no ocupa un lugar destacado en las revistas de contabilidad en lengua española y que hay una carencia importante en revistas especializadas en contabilidad de gestión. Rogério João 
Lunkes, Vicente Ripoll-Feliu y Fabricia Silva da Rosa (2012), de acuerdo a la clasificación temática aportada por James W. Hesford, Sung-Han Lee, Wim A. van der Stede y S. Mark Young (2007) descrita en párrafos anteriores, encontraron en las revistas en lengua española una tendencia casi equilibrada entre las temáticas de contabilidad de costos, de control de gestión y de otras en contabilidad de gestión, a diferencia de las tendencias internacionales, para las que los temas relacionados con la contabilidad de costos no son tan representativos y el control de gestión es casi mayoritario en la producción académica; los otros temas en contabilidad de gestión en las tendencias internacionales son marginales y no son tan representativos como en la producción en lengua española. También concluyen que las publicaciones están centradas mayoritariamente en otros temas de contabilidad de gestión, considerada por las publicaciones internacionales como periférica; esto podría mostrar una falta de enfoque y profundidad de acuerdo con la lógica internacional.

En Iberoamérica, las tendencias no se alejan de lo anteriormente descrito por Rogério João Lunkes, Vicente Ripoll-Feliu y Fabricia Silva da Rosa (2012), pues también están muy cerca las tendencias relacionadas con la contabilidad de costos, control de gestión y otros temas o tópicos en contabilidad de gestión (Salgado, 2011). Incluso, los otros temas de contabilidad de gestión que podrían tomarse como minoría en las tendencias predominantes, en Iberoamérica son los más representativos, esto puede obedecer a la tardía inserción en la contabilidad de gestión como disciplina de inte- rés para investigar, en el afán de replicar las tendencias internacionales emergentes y en la poca importancia otorgada a la contabilidad de costos sobre la contabilidad de gestión y a los asuntos del control organizacional. Los trabajos de revisión son importantes entre los trabajos en Iberoamérica, y hay poca o nula intención de desarrollar nuevas conceptualizaciones (frameworks); esto hace que algunas tendencias se reproduzcan en esfuerzos aislados sin tener programas de investigación estructurados y que se observen de manera parcial e incompleta las tendencias internacionales en contabilidad de gestión. Algunas tendencias, como las relacionadas con la utilización de metodologías cualitativas como el estudio de caso, constituyen un punto importante para destacar en la producción iberoamericana. La producción en contabilidad de gestión en Iberoamérica en comparación con las publicaciones internacionales hegemónicas es casi insignificante; aunque la producción local es creciente, se puede afirmar incluso que los medios de difusión de la producción académica son precarios, a pesar de que el diálogo académico entre países todavía es incipiente y no se refleja de manera importante. Países como España, Brasil, Venezuela y México son los más representativos, lo que muestra el alejamiento de los demás países en la producción académica; en ese sentido, se reclaman más esfuerzos en nuestros entornos en los cuales la contabilidad de gestión no ha calado como una rama importante de estudio en la contabilidad (Salgado, 2011).

En Colombia, los esfuerzos dirigidos a investigar en contabilidad de gestión lucen incipientes; la prevalencia de la contabilidad 
financiera y de la regulación de la contabilidad financiera y el desinterés mostrado hacia la gestión de las organizaciones, pueden configurarse como algunos factores que impiden su amplia difusión y verse incluso reflejados en la dedicación de los investigadores a los temas asociados con la contabilidad de gestión. Algunos investigadores en contabilidad de gestión afirman:

El panorama de la investigación en costos y contabilidad de gestión en Colombia es preocupante, esta área no ocupa un lugar destacado dentro de las ramas de contabilidad que se discuten e investigan en el país, son muy pocos los artículos publicados en las revistas especializadas en temas contables y de gestión, pocos los investigadores que sobresalen en el ámbito nacional, y casi inexistentes las redes de investigadores de diferentes instituciones que discuten el tema (Duque \& Osorio, 2013, p. 39).

Así pues, antes de mostrar un panorama sombrío, la contabilidad de gestión en Colombia muestra un potencial de trabajo extraordinario que paulatinamente puede generar líneas de investigación y de trabajo en las instituciones de educación superior. El diálogo académico y las redes de investigación son fundamentales en esta empresa; en ese sentido, algunos esfuerzos se empiezan a configurar.

\section{Discusiones y conclusiones}

Un conocimiento de las tendencias importantes y adelantos del pasado debería proporcionar al contador moderno una perspectiva contra la cual pudiera evaluar y comparar el pensamiento y las prácticas vigentes (Garner, 1979). La visión histórica proporciona una referencia importante con respecto a los antecedentes disciplinares, a los contextos y tiempos en los que las prácticas de contabilidad de costos y gestión surgen, se desarrollan y se trabajan desde diferentes ámbitos. No es menor la distinción entre el surgimiento como práctica y el desarrollo académico; en alguna porción, la idea del texto subyace al mostrar que las prácticas de contabilidad de costos y gestión surgidas en los contextos empresariales se han trabajado posteriormente en el campo académico; la relación de lo empresarial y lo académico también puede surgir como una discusión que permita vislumbrar la posible separación entre los dos campos. Sin embargo, las temáticas e investigaciones se cruzan en ambos campos. La crisis surgida en los años ochenta del siglo XX (Johnson \& Kaplan, 1988) muestra la desarticulación entre la investigación realizada en contabilidad de gestión y las organizaciones empresariales.

La discusión de lo internacional en contabilidad de gestión estriba en el conocimiento del surgimiento, evolución, tendencias de investigación y prácticas empresariales que dan un panorama de la disciplina en su conjunto. Un error común es tratar la contabilidad de gestión como un conocimiento parcializado, dejando de lado la riqueza de la interrelación disciplinar y la visión completa de la disciplina. El acercamiento hacia lo internacional es un problema de conocimiento, en el cual se debe avanzar, teniendo en cuenta que no se trata de consumir tendencias internacionales sin comprenderlas 
y adaptarlas sin ningún sentido a nuestros contextos. Hay diferencias internacionales sobre cómo se debe orientar la investigación y los esfuerzos académicos en contabilidad de gestión; puede haber críticas a los temas, métodos, incluso a los mismos presupuestos que guían el conocimiento en la contabilidad de gestión. Lo internacional en contabilidad de gestión ayuda a comprender en qué sentido se mueve la academia, en qué direcciones avanzan los investigadores y qué rumbo toman las organizaciones con respecto a las soluciones que la disciplina les ofrece. Incluso si se quieren criticar las tendencias internacionales, deben conocerse muy bien, para poder tener argumentos sólidos sobre sus formulaciones.

La variedad en contabilidad de gestión se puede vislumbrar también en la práctica empresarial. Las diferencias internacionales en las prácticas de contabilidad de gestión son significativas (Amat, Falguera, Puig \& Soldevila, 2003); algunas pueden estar dadas por los objetivos de la contabilidad de gestión, la ideología política, las variables culturales, el entorno empresarial, la legislación fiscal, la legislación en materia de contabilidad de costos y gestión, las organizaciones profesionales contables, la influencia de los países en materia de contabilidad de gestión, el nivel de implantación de técnicas modernas de gestión empresarial y de contabilidad de gestión, el nivel educativo, entre otras razones.

La variedad en los trabajos sobre contabilidad de gestión se mantendrá en el futuro, incluso es posible que aumente, debido a la incorporación de nuevas variantes y combinaciones; entonces, el tipo de conceptualización teórica se seguirá eligiendo y posiblemente cambiando; en definitiva, la conceptualización futura podría ser capaz de aumentar la comprensión de los fenómenos relacionados con la gestión de las organizaciones. Se puede pensar en un enfoque integrador, en el cual los planteamientos económicos se evalúen a la luz de teorías que acerquen mejor a la comunidad investigadora al conocimiento de la compleja realidad empresarial (Escobar \& Lobo, 2002). Podría pensarse que los investigadores seguirán intentando acercarse al conocimiento de las organizaciones de diversas maneras; sin embargo, las tendencias en contabilidad de gestión los seguirán condicionando en gran medida.

Las tendencias internacionales en contabilidad de gestión pueden generar categorías para analizar la producción investigativa en nuestros entornos iberoamericanos. $\mathrm{Al}$ observar las particularidades de nuestros entornos, es importante que no se transfieran las tendencias sin reflexión y crítica; la difusión puede generar el tratamiento de la contabilidad de gestión con un enfoque amplio, diverso y propicio para la integración disciplinar (Salgado, 2010). Las diferencias entre perspectivas internacionales hegemónicas y la dirección que toman las investigaciones en nuestros contextos locales, muestran la reacción de la comunidad académica hacia las tendencias; algunos enfoques estarán dirigidos a replicar las tendencias internacionales; otros estarán analizando otras cuestiones emergentes y poco estudiadas; otros estarán en contra de las tendencias dominantes; otros dentro de la comunidad académica estarán indiferentes. Sin embargo, no hay ninguna razón para desconocer en su integridad el 
entramado disciplinar de la contabilidad de gestión con sus múltiples perspectivas y variantes.

Como su nombre lo indica, la contabilidad de gestión es a su vez gestión y contabilidad. Este razonamiento sería menor si se tuviera más en cuenta que la disciplina contable y la administración de empresas son su fuente de conceptualización; la mirada reducida de la contabilidad de gestión puede llevarla a reducciones en sus conceptualizaciones $y$, por tanto, a una mirada parcial de la riqueza que entraña su entramado disciplinar. Es importante pues, tener un mayor diálogo académico con los administradores de empresas. Este escenario es un tanto utópico, si observamos la poca relación que hay incluso entre los contadores públicos en Colombia; sin embargo, abogar por establecer redes académicas de trabajo conjunto es fundamental para que estas relaciones se den en el futuro. El trabajo investigativo en contabilidad de gestión es una alternativa más que interesante para generar comunidad, una excusa para la reunión, para el trabajo y para la agrupación académica.

\section{Referencias}

\section{Álvarez-López, José \& Blanco-Ibarra, Felipe} (2003). El marco de la contabilidad de gestión. Documentos AECA serie contabilidad de gestión. Madrid: Asociación Española de Contabilidad y Administración de Empresas, AECA.

Amat, Oriol; Falguera, Jordi; Puig, Xavier \& Soldevila, Pilar (2003). Aspectos que influyen en las diferencias internacionales en materia de contabilidad de gestión. Revis- ta Iberoamericana de Contabilidad de Gestión, I (1), 1-16. Disponible en: http://www. observatorio-iberoamericano.org/RICG/ N\%C2\%BA_1/Oriol_Amat_y_otros_autores.pdf

Carmona-Moreno, Salvador (1993). Cambio tecnológico y contabilidad de gestión. Madrid: Ministerio de Economía y Hacienda, Instituto de Contabilidad y Auditoría de Cuentas, ICAC.

Chandler, Alfred Dupont (1977). The Visible Hand: The Managerial Revolution in American Business. Cambridge, Massachusetts, London, England: The Belknap Press of Harvard University Press.

Charnes, Abraham \& Cooper, William W. (1957). Management Models and Industrial Applications of Linear Programming. Management Science, 4 (October), 38-91.

Charnes, Abraham \& Cooper, William W. (1961). Management Models and Industrial Applications of Linear Programming. Vols. 1, 2, New York: John Wiley \& Sons, Inc.

Chatfield, Michael (ed.) (1979). Estudios contemporáneos sobre la evolución del pensamiento contable. México D.F.: Ediciones Contables y Administrativas.

Chua, Wai Fong (1986). Radical Developments in Accounting Thought. The Accounting Review, LXI (4), 601-632.

Dean, Joel (1951). Managerial Economics. Englewood Cliffs, New Jersey: Prentice Hall. Duque-Roldán, María Isabel \& Osorio-Agudelo, Jair Albeiro (2013). Estado actual de la investigación en Costos y contabilidad de gestión en Colombia. Revista del Instituto Internacional de Costos, 11, 26-41. Disponi- 
ble en: http://www.revistaiic.org/articulos/ num11/articulo2_esp.pdf

Escobar-Pérez, Bernabé \& Lobo-Gallardo, Antonio (2002). Implicaciones teóricas y metodológicas de la evolución de la investigación en contabilidad de gestión. Revista Española de Financiación y Contabilidad, REFC, XXXI (111), 245-286. Disponible en: http://www. aeca.es/pub/refc/articulos.php?id=0133

Facin-Lavarda, Carlos Eduardo; Barrachina, Mercedes \& Ripoll-Feliu, Vicente (2008). Paradigmas de investigación en contabilidad de gestión. Revista de Contabilidade do Mestrado em Ciências Contábeis da UERJ, Programa de Mestrado em Ciências Contábeis da Faculdade de Administração e Finanças da Universidade do Estado do Rio Janeiro, XIII (1), 1-18. Disponible en: http://www.epublicacoes.uerj.br/index.php/rcmccuerj/ article/view/5557/4039, http://www.spell. org.br/documentos/download/5

Fayol, Henri (1961). Administración industrial y general. México D.F.: Editorial Herrero Hermanos, Sucesores.

Foster, George \& Young, S. Mark (1997). Frontiers of Management Accounting Research. Journal of Management Accounting Research, 9, 63-77.

Garner, S. Paul (1979). Aspectos relevantes en el desarrollo de la contabilidad de costos. En Michael Chatfield (ed.). Estudios contemporáneos sobre la evolución del pensamiento contable, 247-259. México D. F.: Ediciones Contables y Administrativas.

Gutiérrez-Hidalgo, Fernando (2005). Evolución histórica de la contabilidad de costes y de gestión (1885-2005). De Computis, Re- vista Española de Historia de la Contabilidad, 2, 100-122. Disponible en: http://www.decomputis.org/dc/articulos_doctrinales/gutierrez2.pdf

Hernández-Esteves, Esteban (2006). La historia de la contabilidad: una disciplina en auge. Revista Internacional Legis de Contabilidad y Auditoría, 25, 191-213.

Hesford, James W.; Lee, Sung-Han; Stede, Wim A. van der \& Young, S. Mark (2007). Management Accounting: a Bibliographic Study. En Christopher S. Chapman, Anthony G. Hopwood \& Michael D. Shields (eds.). Handbook of Management Accounting Research, 3-26. Oxford: Elsevier.

Hopwood, Anthony G. (1987). The Archaeology of Accounting Systems. Accounting, Organizations and Society, 12 (3), 207-234.

Jackson, J. Hugh (1979). Medio siglo de progreso en la contabilidad de costos. En Michael Chatfield (ed.). Estudios contemporáneos sobre la evolución del pensamiento contable, 261-277. México D. F.: Ediciones Contables y Administrativas.

Johnson, H. Thomas (1972). Early Costs Accounting for Internal Management Control: Lyman Mills in the 1850's. Business History Review, 46 (4), 466-474. doi:10.2307/3113343.

Johnson, H. Thomas (1981). Toward a New Understanding of Nineteenth Century Costs Accounting. Accounting Review, 56 (3), 510518.

Johnson, H. Thomas \& Kaplan, Robert S. (1988). La contabilidad de costes. Auge y caída de la contabilidad de gestión. Barcelona: Plaza \& Janés. 
Kaplan, Robert S. (1984). The Evolution of Management Accounting. The Accounting Review, 59 (3), 390-418. Disponible en: http://coin.wne.uw.edu.pl/ pmodzelewski/ The\%20Evolution\%20of\%20

Management\%20Accounting.pdf

Kaplan, Robert S. (1988). One Cost System Isn't Enough. Harvard Business Review, 66 (1), 61-66. Disponible en: https://hbr. org/1988/01/one-cost-system-isnt-enough

Kaplan, Robert S. (1993). La evolución de la contabilidad de gestión. En Salvador Carmona-Moreno (ed.). Cambio tecnológico y contabilidad de gestión. Madrid: Instituto de Contabilidad y Auditoría de Cuentas, ICAC, Ministerio de Economía y Hacienda.

Kaplan, Robert S. \& Johnson, H. Thomas (1987). Relevance Lost: The Rise and Fall of Management Accounting. Boston: Harvard Business School Press.

Larrinaga-González, Carlos (1999). Perspectivas alternativas de investigación en contabilidad: una revisión. Revista de contabilidad, 2 (3), 103-131. Disponible en: http://www. rc-sar.es/verPdf.php?articleId $=45$

Lukka, Kari (2010). The Roles and Effects of Paradigms in Accounting Research. Management Accounting Research, 21 (2), 110-115.

Lunkes, Rogério João; Ripoll-Feliu, Vicente \& Silva da Rosa, Fabricia (2012). Un estudio de artículos sobre contabilidad de gestión en revistas de lengua española. Revista Iberoamericana de Contabilidad de Gestión, $X$ (19), 1-12. Disponible en: http://www. observatorio-iberoamericano.org/RICG/ N\%C2\%BA_19/Rogerio_Joao_Lunkes;_Vi-
cente_Ripoll_y_Fabricia_Silva_da_Rosa. pdf

Mattessich, Richard (2001). Hitos de la investigación en contabilidad moderna (segunda mitad del siglo). Revista Legis del Contador, 6, 9-25.

Pasdermadjian, Hrant (1947). Le gouvernement des grandes organisations. Paris: Presses Universitaires de France, PUF.

Ripoll-Feliu, Vicente (coord.) (1994). Introducción a la contabilidad de gestión. Cálculo de costes. Madrid: McGraw-Hill.

Ryan, Bob; Scapens, Robert W. \& Theobald, Michael (2004). Metodología de la investigación en finanzas y contabilidad. Barcelona: Deusto.

Salgado-Castillo, Jorge Andrés (2010). Aproximación general para el análisis de tendencias en la contabilidad de gestión en Iberoamérica. Cuadernos de Contabilidad, 11 (28), 19-40. Disponible en: http://revistas. javeriana.edu.co/index.php/cuacont/article/ view/3175/2415

Salgado-Castillo, Jorge Andrés (2011). Tendencias de investigación en contabilidad de gestión en Iberoamérica (1998-2008). Cuadernos de contabilidad, 12 (30), 273305. Disponible en: http://revistas. javeriana.edu.co/index.php/cuacont/article/ view/3120/2292

Solomons, David (1968). The Historical Development of Costing. En David Solomons (ed.). Studies in Costs Analysis, 3-49. Homewood, Illinois: Richard D. Irwin.

Vaes, Urbain (1952). La Hiérarchie dans la structure de l'entreprise. Paris: Dunod. 
TENDENCIAS EN CONTABILIDAD DE GESTIÓN: UNA MIRADA A SU EVOLUCIÓN / J. SALGADO / 805

Vlaemminck, Joseph-H. (1961). Historia y doctrinas de la contabilidad. Madrid: EJES.

Watts, Ross L. \& Zimmerman, Jerold (1978, January). Towards a Positive Theory of the Determination of Accounting Standards. The Accounting Review, 53 (1), 112-134. Disponible en: http://marriottschool.net/emp/tc259/accframework/ files/1978\%20Watts\%20and\%20Zimmerman.pdf

Watts, Ross L. \& Zimmerman, Jerold (1979, April). The Demand for and Supply of Accounting Theories: the Market for Excuses. The Accounting Review, 54 (2), 273 305.
Watts, Ross L. \& Zimmerman, Jerold (1986). Positive Accounting Theory. Englewood Cliffs, New Jersey: Prentice Hall.

- Fecha de recepción: 1 de julio de 2014

- Fecha de aceptación: 6 de octubre de 2014

- Disponible en línea: 15 de diciembre de 2014

\section{Para citar este artículo}

Salgado-Castillo, Jorge Andrés (2014). Tendencias en contabilidad de gestión: una mirada a su evolución (finales del siglo XIX y siglo XX). Cuadernos de Contabilidad, 15 (39), 787-805.

doi: 10.11144/Javeriana.cc15-39.tcgm 
